\title{
Análise da escala de valores organizacionais em empresas com estrutura de gestão
}

\section{familiar}

\author{
Analysis of the scale of organizational values in companies with a family management structure \\ Análisis de la escala de valores organizacionales en empresas con estructura de gestión familiar
}

Recebido: 25/04/2021 | Revisado: 03/05/2021 | Aceito: 08/05/2021 | Publicado: 26/05/2021

Luciana Silva Fonseca

ORCID: https://orcid.org/0000-0001-5588-7006 Universidade Federal Fluminense, Brasil

E-mail: luc.fonseca@uol.com.br

Denise Medeiros Ribeiro Salles

ORCID: https://orcid.org/0000-0001-8378-7117 Universidade Federal Fluminense, Brasil

E-mail: denisesalles@id.uff.br

João Alberto Neves dos Santos

ORCID: https://orcid.org/0000-0002-4812-6214

Universidade Federal Fluminense, Brasil E-mail: joaoneves@id.uff.br

Andrey Pimentel Aleluia Freitas

ORCID: https://orcid.org/0000-0003-0136-5696

Universidade Federal Fluminense, Brasil

E-mail: andreyfreitas@id.uff.br

\begin{abstract}
Resumo
Os valores organizacionais funcionam como parâmetros para a avaliação de comportamento, conduta e motivação profissional. São elementos essenciais para a percepção e o entendimento da cultura organizacional, sobretudo em empresas com estrutura de gestão familiar. Com base no modelo da Escala de Valores Organizacionais - EVO, este artigo objetivou verificar a adequação do modelo a profissionais que atuam em empresas com estrutura de gestão familiar. Para isso, essa Escala foi aplicada em uma amostra composta por 360 profissionais atuantes em empresas familiares, de médio e grande portes, no estado do Rio de Janeiro, nos segmentos de comércio, indústria e serviços. A avaliaçao foi conduzida por meio de técnicas de análise fatorial, necessárias para validar e testar o modelo estrutural. Procedimentos estatísticos foram utilizados para descrever a amostra quanto as propriedades de semelhança entre suas variáveis. Os resultados obtidos identificaram conjuntos de variáveis que configuram um modelo adaptado da estrutura de valores organizacionais em empresas familiares, além de escalas de ajustes necessários para confirmar aderência do modelo adaptado ao modelo original da EVO da amostra delimitada. Com isso, foi possível responder ao questionamento central desta pesquisa, indicando que o modelo original, caracterizado 38 variáveis distribuídas em 5 fatores, resultou em um modelo adaptado, caracterizado por significância estística $(\mathrm{p}<0,05)$, consistência e qualidade dos índices $\left(\mathrm{X}^{2} / \mathrm{gl}=2,146\right.$, GFI $=0,942$, AGFI $=0,909, \mathrm{CFI}=0,948$, RMSEA $\left.(90 \% \mathrm{IC})=0,056\right)$ obtidos pelos ajustes, configurando uma nova estrutura com 16 variáveis, distribuídas nos 5 fatores do modelo original da EVO.

Palavras-chave: Escala de Valores Organizacionais - EVO; Cultura organizacional; Empresas familiares; Análise fatorial.
\end{abstract}

\begin{abstract}
The organizational values serve as parameters for the assessment of behavior, conduct and professional motivation. They are essential elements for the perception and understanding of the organizational culture, especially in companies with a family management structure. Based on the Organizational Values Scale - EVO model, this article aimed to verify the adequacy of the model to professionals working in companies with a family management structure. For this, this Scale was applied to a sample composed of 360 professionals working in family businesses, medium and large, in the state of Rio de Janeiro, in the segments of commerce, industry and services. The evaluation was conducted by means of factor analysis techniques, necessary to validate and test the structural model. Statistical procedures were used to describe the sample regarding the similarity properties between its variables. The results obtained identified sets of variables that configure a model adapted from the structure of organizational values in family businesses, in addition to scales of adjustments necessary to confirm adherence of the model adapted to the original EVO model of the delimited sample. With that, it was possible to answer the central questioning of this research, indicating that the original model, characterized by 38 variables distributed in 5 factors, resulted in an adapted model, characterized by statistical significance $(\mathrm{p}<0.05)$, consistency and quality of the indices $\left(\mathrm{X}^{2} / \mathrm{gl}=\right.$
\end{abstract}


2,146, GFI $=0,942, \mathrm{AGFI}=0,909, \mathrm{CFI}=0,948, \operatorname{RMSEA}(90 \% \mathrm{IC})=0,056)$ obtained by the adjustments, configuring a new structure with 16 variables, distributed in the 5 factors of the original EVO model.

Keywords: Scale of Organizational Values - EVO; Organizational culture; Family businesses; Factor analysis.

\section{Resumen}

Los valores organizacionales actúan como parámetros para la evaluación del comportamiento, la conducta y la motivación profesional. Son elementos esenciales para la percepción y comprensión de la cultura organizacional, especialmente en empresas con estructura de gestión familiar. Con base en el modelo Escala de Valores Organizacionales - EVO, este artículo tuvo como objetivo verificar la adecuación del modelo a los profesionales que trabajan en empresas con estructura de gestión familiar. Para ello, esta Escala se aplicó a una muestra compuesta por 360 profesionales que laboran en empresas familiares, medianas y grandes, en el estado de Río de Janeiro, en los segmentos de comercio, industria y servicios. La evaluación se realizó mediante técnicas de análisis factorial, necesarias para validar y probar el modelo estructural. Se utilizaron procedimientos estadísticos para describir la muestra con respecto a las propiedades de similitud entre sus variables. Los resultados obtenidos identificaron conjuntos de variables que configuran un modelo adaptado de la estructura de valores organizacionales en empresas familiares, además de escalas de ajustes necesarios para confirmar la adherencia del modelo adaptado al modelo EVO original de la muestra delimitada. Con ello, fue posible responder al cuestionamiento central de esta investigación, indicando que el modelo original, caracterizado por 38 variables distribuidas en 5 factores, resultó en un modelo adaptado, caracterizado por significancia estadística $(\mathrm{p}<0.05)$, consistencia y calidad de los índices $\left(\mathrm{X}^{2} / \mathrm{gl}=2,146\right.$, $\mathrm{GFI}=0,942, \mathrm{AGFI}=0,909, \mathrm{CFI}=0,948, \mathrm{RMSEA}(90 \% \mathrm{IC})=0,056)$ obtenidos por los ajustes, configurando una nueva estructura con 16 variables, distribuidas en los 5 factores del modelo EVO original.

Palabras clave: Escala de Valores Organizacionales - EVO; Cultura de la organización; Empresas familiares; Análisis factorial.

\section{Introdução}

A análise da cultura de organizações bem-sucedidas permite identificar pontos em comum ligados as estratégias que essas organizações implementam para fundamentar seus valores e alcançar seus objetivos. Segundo Tamayo e Gondim (1996) os valores são constitutivos da própria cultura e seu conhecimento por parte da organização está ligado a estratégias e políticas que promovem o desenvolvimento organizacional.

Os valores envolvem tudo o que é importante para os indivíduos. São conceitos estruturados, que norteiam e interferem em vários aspectos da vida: julgamentos morais, reações pessoais às situações sociais, compromissos com metas pessoais e organizacionais. Silenciosamente, os valores atuam naturalmente, direcionando as decisões tomadas nos diversos níveis que configuram a organização. Dessa forma, os líderes, principalmente os seniores, estruturam a base da cultura característica da organização (Chukwuka, 2016).

Considerando a cultura como um fator presente nas diferentes interações entre os indivíduos, entende-se que, em uma organização, a cultura é a percepção coletiva que os diferentes membros têm da organização. Essa percepção resulta em formas de administrar, projetos e posições de mercados diferenciados. Nas organizações familiares a cultura integra valores familiares e do negócio (Arayesh et al., 2017). Além disso, de acordo com Chukwuka (2016), a cultura organizacional representa um sistema de valores que cria um sentimento de identidade nos membros da organização e os direciona para atingir objetivos em comum.

Para Schein (2009) a cultura organizacional é preponderante para o entendimento da performance de profissionais e da própria organização. Assim, seu estudo permite a identificação de forças e fraquezas de uma empresa, podendo influenciar o grau de impacto das decisões estratégicas (Arayesh et al., 2017).

A quantidade de empresas controladas por estruturas de gestão familiar é significativa, refletindo na relevância desse aspecto para a configuração da economia mundial. Esse ponto realça a real dimensão do capital humano para a evolução das organizações, sobretudo as que apresentam estrutura de gestão familiar.

Com o objetivo de abordar a relação entre a Escala de Valores Organizacionais - EVO de Tamayo e Godim (1996) e os valores característicos de profissionais inseridos em ambientes organizacionais com gestão familiar, este trabalho apresenta 
uma abordagem quantitativa, a partir da utilização de um instrumento de pesquisa validado: a Escala de Valores Organizacionais - EVO. Sua aplicação é direcionada a uma amostragem não probabilística, caracterizada por 360 profissionais de empresas familiares, localizadas no estado do Rio de Janeiro e presentes nos segmentos da indústria, do comércio e de serviços. Por meio dessa abordagem será possível responder ao questionamento central da pesquisa:

Q: Quais valores organizacionais estão presentes em empresas com estrutura de gestão familiar de acordo com o modelo teórico da Escala de Valores Organizacionais - EVO?

A condução da pesquisa seguiu por meio da aplicação de conceitos estatísticos multivariados, por meio dos softwares IBM SPSS ${ }^{\circledR}$ V.21 e IBM AMOS ${ }^{\circledR}$ V.21. Inicialmente foi utilizada a análise fatorial exploratória (AFE) para identificar o grau de correlação presente entre as cargas fatoriais das variáveis estudadas. Em seguida, com base nos fundamentos da análise fatorial confirmatória (AFC), foi realizado o comparativo entre os modelos original e adaptado da EVO, verificando se a qualidade dos ajustes propostos pelo modelo adaptado atingiu valores consistentes e satisfatórios ao modelo original da EVO.

\section{Fundamentação teórica}

\subsection{Valores organizacionais}

Intuitivamente, valores são definidos como algo importante, cuja violação pode provocar sentimento de culpa. Valores são descritos na literatura como crenças que motivam ações e situações, orientam e avaliam, priorizando eventos, conforme seus níveis de importância.

Os valores organizacionais são relacionados de forma positiva ao desempenho alcançado no trabalho. Nesse sentido, muitas pesquisas fazem associações a essa relação, destacando temas como compromisso, cultura organizacional, satisfação no trabalho, engajamento, trabalho em equipe, integração e aprendizagem organizacional (Chukwuka, 2016).

A natureza estável, porém, dinâmica dos valores, os torna a base do comportamento e da identidade dos indivíduos. Esse aspecto evidencia sua influência nas respostas comportamentais e na conduta social dos indivíduos, nos diversos ambientes onde interagem. Quando isso se desenvolve no ambiente de trabalho, faz com que os indivíduos passem a alinhar seus valores aos valores organizacionais (Schwartz, 2019).

Para Tamayo e Gondim (1996), os valores são estruturados por concepções cognitivas de necessidades e razões. Os valores adquiridos através das experiências vividas compõem o conjunto de atitudes que norteiam as atitudes comportamentais (Lewis, 1991; Rokeach, 1981; Schwartz, 2019).

De forma similar aos indivíduos, as organizações possuem valores que norteiam seus processos e posicionamento dos seus integrantes. Esses valores organizacionais cooperam com a integração interna, fomentando o alcance de objetivos e metas que são inevitáveis para o atingimento da eficácia empresarial.

O interesse sobre temas relacionados a valores organizacionais teve considerável crescimento nos últimos anos. Uma explicação plausível para isso pode ser fundamentada pela relevância do seu papel para a compreensão da cultura (Carvalho; Oliveira; Silva, 2013). Os valores são compartilhados por grande parte dos membros e possuem missão integradora no ambiente organizacional (Oliveira, 2004; Tamayo \& Gondim, 1996).

Veiga (2018) enfatiza que o conceito de valor organizacional não pode ser limitado somente à interação entre os integrantes de uma organização. O valor de uma organização tem forte ligação com a imagem dos seus produtos, seu compromisso com diversas frentes de atuação e com a lisura a ser apresentada para o mercado.

\subsection{Cultura organizacional}

A definição de cultura de uma organização passa pelo entendimento da sua personalidade e do seu caráter, compreendendo seus valores, crenças e pressupostos. Norteia o comportamento, as interações, a forma como as decisões 
devem ser tomadas e como as atividades de trabalho devem ser realizadas por seus colaboradores.

Cada organização está ligada à sua cultura, compreendendo valores, normas, regulamentos, sistemas de crenças e códigos de conduta, para direcionar suas operações e orientar o comportamento dos seus membros.

Para Chukwuka (2016), a cultura organizacional segue uma planificação mental coletiva que distingue grupos, resultando em contínuos processos de aprendizagem. Essa compreensão coletiva direciona que os membros de uma determinada organização apresentem comportamentos e visões diferente de outros, fazendo com que as formas de gestão, condução de projetos e posições de mercado sejam conduzidos de maneiras particularmente diferenciadas (Russo, 2012).

De uma forma geral, o conceito de cultura ajuda as pessoas a entenderem o que está oculto nos comportamentos. Dessa forma, essa abordagem induz à percepção de que a cultura de uma organização pode ser um atributo profundamente enraizado em suas diferentes estruturas (Motta \& Caldas, 1997; Zaneti et al, 2014).

Os conceitos-chave da cultura de uma organização contemplam sua história, o ambiente em que está inserida e como seus líderes conduzem suas equipes para o propósito dela. Dessa forma, sua compreensão é fundamental para a eficácia da sua liderança. Por meio da compreensão da cultura organizacional, os gestores assumem melhores posições para implementar estratégias e atingir suas metas.

Muitas vezes é difícil especificar o que exatamente impulsiona uma cultura particular, mas é mais fácil observar seus efeitos. Por exemplo, uma pequena empresa pode apresentar cultura bem diferente de uma empresa de grande porte que, por sua vez, é diferente da cultura de um hospital. Dessa forma, pode levar anos para que os novos colaboradores entendam e absorvam uma cultura organizacional por completa (Ouchi, 1982; Maximiano; 2005; Lacerda, 2011).

Para obter uma melhor compreensão da cultura de uma organização, suas regras escritas e não escritas devem ser examinadas juntamente com os relacionamentos, valores e comportamentos exibidos por seus indivíduos. Esta lista de verificação inicia-se com o delineamento das dimensões da cultura organizacional.

Nenhuma organização consegue atingir seus propósitos sem que haja interação e contribuição guiadas, especialmente nos aspectos associados aos níveis de relacionamento entre seus colaboradores e a gestão. Nesse sentido, as organizações utilizam esse relacionamento para incutir nos funcionários a importância do comprometimento com seus valores e fazê-los sentirem-se pertencentes ao todo.

\subsection{Escala de valores organizacionais}

Os valores organizacionais são geralmente definidos como concepções mentais, associadas a princípios que orientam práticas e referências organizacionais. São comuns a indivíduos que vivem e compartilham do mesmo ambiente organizacional, organizado hierarquicamente. Embora estejam no nível consciente, são mais abrangentes do que as próprias práticas organizacionais.

Não é fácil definir os valores principais dentro da cultura organizacional, nem como podem ser matematicamente estruturados para configurar, de forma ampla, os valores organizacionais. Dessa forma, este artigo considera a Escala de Valores Organizacionais - EVO de Tamayo e Gondim (1996), por esta ser uma das escalas de valores organizacionais mais citadas nas literaturas nacional e internacional.

Tamayo e Gondim (1996) estruturaram os valores organizacionais a partir das interpretações motivacional, cognitiva e estruturante. $\mathrm{O}$ aspecto motivacional relaciona os valores às metas e objetivos conscientes das organizações. No aspecto cognitivo dos valores são consideradas as concepções que são, ou não, desejáveis pelas organizações.

Para estimar o delineamento dos valores organizacionais, os autores consideraram 38 itens, cada um associado a um valor organizacional. Por meio da abordagem estatística, a validação fatorial identificou cinco grupos, também conhecidos por fatores ou variáveis latentes: 
a) O grupo inicial, associado a eficácia/eficiência, apresentou nove itens em sua estrutura (eficiência, eficácia, produtividade, qualidade, planejamento, comprometimento, competência, dedicação e pontualidade), que avaliam a percepção dos indivíduos da prioridade que as empresas dedicam a cada um desses valores;

b) O segundo grupo, associado a interação no trabalho, consiste em dez itens em sua estrutura (coleguismo, abertura, cooperação, amizade, democracia, criatividade, flexibilidade, benefícios, sociabilidade e competitividade), que medem o quanto a organização valoriza a interação positiva nos postos de trabalho, sob a ótica dos colaboradores;

c) O terceiro grupo, que representa aspectos de gestão, compreende sete itens em sua estrutura (hierarquia, tradição, fiscalização, obediência, organização, supervisão e postura profissional), avaliando o quanto os valores da gestão, sob o conceito tradicional e hierárquico, são considerados pela organização no entendimento dos colaboradores;

d) O quarto grupo está relacionado com a inovação e é caracterizado por quatro itens (integração inter organizacional, incentivo à pesquisa, probidade e modernização dos recursos), que indicam o quanto a organização valoriza os aspectos inovadores, sobre o entendimento dos colaboradores;

e) O quinto grupo, definido como respeito ao servidor, consiste em oito itens (reconhecimento do mérito, respeito, polidez, qualificação dos recursos humanos, honestidade, justiça, harmonia e plano de carreira) indicam o quanto a organização valoriza o respeito aos funcionários, na visão dos seus colaboradores.

\subsection{Empresas de gestão familiar}

Um dos principais desafios enfrentados por pesquisas direcionadas a empresas familiares consiste na definição do que exatamente caracteriza uma empresa familiar, distinguindo-a das não familiares. A maioria das pesquisas é fundamentada por conceitos e escolhas de suporte empírico. Além disso, a literatura mostra que poucos países têm informações explícitas em seus bancos de dados para capacitá-los a reconhecer empresas familiares.

Vários autores tentam identificar as qualidades intrínsecas e a natureza fundamental dos corpos estranhos. Arif et al. (2017) vêm a essência de um corpo estranho, como uma visão desenvolvida por uma forte aliança controlada por indivíduos da mesma família, ou por um pequeno grupo de famílias, mantido ao longo de gerações. Arayesh et al (2017) acreditam que a essência deve incluir o conceito de família. Os autores sugerem que as empresas influenciadas por famílias diferem de outras empresas por possuírem um conjunto único de recursos e capacidades resultantes das interações entre a unidade familiar, a entidade empresarial e os membros individuais da família (Arayesh et al, 2017). Essas definições teóricas devem ser transformadas em definições operacionais para a realização de estudos empíricos. No entanto, as definições operacionais têm suas próprias limitações.

Alguns autores baseiam sua definição em critérios objetivos, como a porcentagem de propriedade familiar ou o número de membros da família que ocupam cargos de gerência ou conselho (Arif et al., 2017). Outros pesquisadores definem uma empresa como familiar com base em aspectos subjetivos, direcionados pela intenção de transferir a propriedade para a próxima geração como um critério.

Como a existência de componentes torna a essência possível, algumas pesquisas consideram o envolvimento da família para definir o caráter da organização, dentro de um termo amplo que inclui propriedade familiar, gestão, governança e continuidade de sucessão transgeracional (Arif et al.,2017).

O desempenho é um indicador essencial do sucesso organizacional e da vantagem competitiva das organizações. Se as empresas são capazes de identificar os fatores que determinam melhorias no desempenho, podem tirar proveito de seus atributos particulares. Nesse caso, os aspectos relacionados à cultura familiar podem ser disseminados como forma de envolver 
o quadro de funcionários com os valores da organização, incentivando relações, crenças e percepções similares ao ambiente familiar (Arayesh et al, 2017).

No cenário nacional, segundo dados da Arayesh et al (2017), quatro organizações de estrutura familiar estão entre o grupo das dez maiores empresas brasileiras. Na Europa, os associados da European Family Business, entidade que reúne as principais empresas familiares do continente, respondem por pelo menos 9\% do PIB do bloco econômico. Estes dados evidenciam a importância deste tipo de estrutura de organização para a economia mundial.

Mas qual aspecto transforma empresas familiares em um tipo especial de organização? Os proprietários de empresas familiares sabem que exercem papel diferenciado das empresas com outras características. Os funcionários das empresas familiares conhecem a importância dos laços de família no ambiente de negócios, na cultura da empresa e em suas carreiras. Os profissionais de marketing consideram a imagem da empresa familiar como vantagem junto aos clientes. E as famílias entendem que a união, dento e fora da organização, é importante em suas vidas.

Para muitas pessoas, os dois fatores de maior relevância em suas vidas são a família e o trabalho. E buscam organizações que valorizam esses aspectos. A empresas com gestão familiar configuram uma estrutura organizacional diferenciada, cujo "caráter especial" pode provocar diferentes impactos nos contextos profissional ou afetivo.

A história da organização familiar, enquanto empresa, junto com sua identidade e a linguagem comum das famílias pode desenvolver e potencializar interações entre seus componentes. Quando os dirigentes são parentes, alguns conceitos, interesses e valores surgem de uma fonte comum e a comunicação pode ser instantânea e dinâmica. Entretanto, esta mesma proximidade pode comprometer os aspectos profissionais, muitas vezes fundamentais para a correta condução do ambiente organizacional.

Antigas histórias da família podem refletir diretamente nas relações dentro da organização. Exercer autoridade com parentes, nem sempre é fácil. Os papéis na família e na empresa podem tornar-se confusos ou invertidos. As pressões do ambiente de trabalho podem sobrecarregar e desgastar relacionamentos profissionais e, em alguns casos, até relacionamentos afetivos.

O cenário globalizado e a internacionalização dos mercados fizeram com que muitas empresas familiares identificassem a necessidade de transição de suas gestões. As pequenas e médias empresas, mesmo eficientes e eficazes, passaram a buscar, com urgência, novos patamares de competitividade para manterem-se sólidas no mercado. Para isso, passaram a valorizar seus profissionais e retê-los, de modo a desenvolver neles os mesmos níveis de relacionamento presentes na família.

Nesse contexto insere-se o interesse desta pesquisa, verificando se os laços desenvolvidos entre a gestão e os funcionários tornam perceptíveis os valores da organização familiar, contagiando e incentivando todos em um ambiente competitivo, de forma similar aos valores propostos por empresas com outras formas de estrutura

\section{Metodologia}

\subsection{Caracterização da amostra}

Considerando que a amostra do estudo foi definida para representar uma população da qual esse grupo pertence e que, devido ao não conhecimento da real dimensão de quantos profissionais estão envolvidos com empresas com estrutura de gestão familiar, seria custoso, demorado ou inviável identificar sua totalidade. Por esse motivo, optou-se por uma amostragem nãoprobabilística por conveniência, caracterizada pela escolha de profissionais que trabalham em organizações com estrutura familiar situadas no estado do Rio de Janeiro.

A amostragem não probabilística por conveniência é indicada quando há facilidade de acesso dos pesquisadores às empresas, aos seus funcionários, proximidade geográfica (todas no estado do Rio de Janeiro) e aos aspectos relacionados à 
disponibilidade e vontade dos participantes em responder (Farrokhi \& Mahmoudi-Hamidabad, 2012; Marôco, 2014).

A amostra foi caracterizada por 360 profissionais que trabalham há mais de três anos em empresas de médio e grande portes, com estruturas de administração familiar, nos segmentos de comércio, indústria e serviços. Sob esse modelo, o tamanho sugerido para a amostra deve ser superior a 100 observações, considerando ainda que essa quantidade de amostras corresponda a, pelo menos, cinco vezes do número de variáveis utilizadas pelo instrumento de pesquisa (HAIR et al., 2014).

A aplicação do questionário Escala de Valores Organizacionais - EVO foi realizada de forma presencial nas empresas. O questionário foi aplicado a 360 profissionais, distribuídos da seguinte forma: 120 funcionários de empresas do segmento de serviços, 120 funcionários de empresas do segmento de comércio e 120 funcionários do segmento da indústria. Foram selecionadas empresas de gestão familiar, com o mínimo de 10 anos no mercado.

A Escala de Valores Organizacionais - EVO é um instrumento de pesquisa estruturado, composto por 38 variáveis alocadas em cinco grupos. As respostas elaboradas são caracterizadas por uma escala do tipo Likert, com estrutura fechada. Importante destacar que o uso de escalas confere simplicidade e objetividade para as respostas contidas no instrumento (Gil, 1999). O Quadro 1 representa o modelo de escala Likert adotado por esta pesquisa.

Quadro 1: Identificação do fator de carregamento em função do tamanho da amostra.

\begin{tabular}{|c|c|c|c|c|}
\hline Nada importante & Pouco importante & Mais ou menos importante & Importante & Extremamente importante \\
\hline 0 & 1 & 2 & 3 & 4 \\
\hline
\end{tabular}

Fonte: Autores (2021).

A escala adotada propõe que as respostas sejam classificadas por uma variação de cinco pontos, indo de zero a quatro (0-4), onde o menor valor indica a irrelevância do grau de percepção dos respondentes e o maior valor, sua extrema consideração. Assim, na Escala de Valores Organizacionais (EVO), o zero significa que o valor não é relevante como um princípio orientador dos valores da organização. Quanto maior for o número da resposta, maior relevância terá o valor, pelo princípio orientador dos valores organizacionais.

A apuração dos resultados produz um perfil de escores composto pelos cinco grupos, ou variáveis latentes, da EVO. O Quadro 2 representa a distribuição dos itens utilizados para a avaliação dos escores de cada grupo. 
Quadro 2: Itens para apuração dos resultados da EVO.

\begin{tabular}{|c|c|c|}
\hline Grupo & Item & \begin{tabular}{|l|} 
Descrição do Item \\
\end{tabular} \\
\hline \multirow{9}{*}{ Eficácia - Eficiência } & 5 & Competência \\
\hline & 7 & Comprometimento \\
\hline & 10 & Dedicação \\
\hline & 12 & Eficácia \\
\hline & 13 & Eficiência \\
\hline & 25 & Planejamento \\
\hline & 28 & Pontualidade \\
\hline & 31 & \begin{tabular}{|l|} 
Produtividade \\
\end{tabular} \\
\hline & 32 & Qualidade \\
\hline \multirow{10}{*}{ Interação no Trabalho } & 1 & Abertura \\
\hline & 2 & Amizade \\
\hline & 3 & Benefícios \\
\hline & 4 & Coleguismo \\
\hline & 6 & Competitividade \\
\hline & 8 & Cooperação \\
\hline & 9 & Criatividade \\
\hline & 11 & Democracia \\
\hline & 15 & Flexibilidade \\
\hline & 36 & Sociabilidade \\
\hline \multirow{7}{*}{ Gestão } & 14 & Fiscalização \\
\hline & 17 & Hierarquia \\
\hline & 23 & Obediência \\
\hline & 24 & Organização \\
\hline & 29 & \begin{tabular}{|l} 
Postura Profissional \\
\end{tabular} \\
\hline & 37 & Supervisão \\
\hline & 38 & Tradição \\
\hline \multirow{4}{*}{ Inovação } & 19 & Incentivo à Pesquisa \\
\hline & 20 & Integração \\
\hline & 22 & Modernização \\
\hline & 30 & \begin{tabular}{|l|} 
Probidade \\
\end{tabular} \\
\hline \multirow{8}{*}{ Respeito ao Funcionário } & 16 & Harmonia \\
\hline & 18 & Honestidade \\
\hline & 21 & Justiça \\
\hline & 26 & Plano de Carreira \\
\hline & 27 & Polidez \\
\hline & 33 & Qualificação RH \\
\hline & 34 & Reconhecimento \\
\hline & 35 & Respeito \\
\hline
\end{tabular}

Fonte: Tamayo e Gondim (1996).

\subsection{Análise preliminar dos dados}

Os procedimentos estatísticos devem iniciar a partir da verificação da distribuição e da validade dos dados. Nesse sentido, os dados foram verificados e constatou-se que os dados apresentavam distribuição normal ( $<<0,05)$, resultando assim, em rejeição da hipótese nula (H0: Os dados não apresentam distribuição normal).

A partir da análise da distribuição dos dados, premissa essencial na condução da análise fatorial multivariada, verificou-se os níveis de validade das respostas e seus respectivos níveis de representatividade. Nesse sentido, a consistência interna do instrumento deve ser considerada como referência da confiabilidade da estrutura do questionário, cuja representação induz ao entendimento de nível de distanciamento que os itens associam a um mesmo conceito. A validade é geralmente percebida a partir das validações do conteúdo e do constructo (Nunnally, 1967).

Com base nos resultados encontrados nos testes Kaiser-Meyer-Olkmin (KMO), do coeficiente Alfa de Cronbach e de esfericidade de Bartlett foram verificadas, de modo respectivo, a validade de conteúdo, a confiabilidade e a objetividade do 
instrumento de pesquisa (Field, 2020). Os resultados são mostrados pela Tabela 1.

Tabela 1: Resumo do processamento do questionário e da confiabilidade na frequência dos atrasos.

\begin{tabular}{|c|c|c|c|c|}
\hline \multicolumn{4}{|c|}{ Resumo de Processamento de Casos } & \multirow{4}{*}{$\begin{array}{r}100 \% \\
0 \% \\
100 \%\end{array}$} \\
\hline \multirow{3}{*}{ Casos } & Válidos & 360 & & \\
\hline & Excluídos & 0 & & \\
\hline & Total & 360 & & \\
\hline \multicolumn{5}{|c|}{ Estatísticas de confiabilidade } \\
\hline \multirow{3}{*}{\multicolumn{2}{|c|}{$\begin{array}{r}\text { Alfa de Cronbach } \\
\text { Alfa de Cronbach com base em itens padronizados } \\
N \text { Total de Itens }\end{array}$}} & & 0,935 & \\
\hline & & & 0,937 & \\
\hline & & & 38 & \\
\hline \multicolumn{5}{|c|}{ Testes de KMO e Bartlett } \\
\hline \multicolumn{4}{|c|}{ Medida Kaiser-Meyer-Olkmin de adequação de amostragem } & 0,912 \\
\hline \multirow{3}{*}{\multicolumn{2}{|c|}{ Teste de esfericidade de Bartlett }} & Aprox. Qui-quadrado & & 5959,114 \\
\hline & & $g l$ & & 703 \\
\hline & & Sig & & 0,000 \\
\hline
\end{tabular}

Fonte: Autores (2021).

De acordo com a Tabela 1 verifica-se um excelente valor para o Alfa de Cronbach $(\alpha=0,937)$, significando elevada consistência interna pela escala de confiabilidade utilizada, conforme destaca a literatura. A aplicação do teste de KaiserMeyer- Olkmin $(\mathrm{KMO})$ verificou a efetividade da amostra definida $(\mathrm{KMO}=0,912)$, apresentando excelente resultado. A utilização do teste de esfericidade de Bartlett, que objetiva verificar o nível de correlação entre as variáveis, onde seu resultado conferiu elevado grau de significância estatística, rejeitando a configuração de uma matriz identidade.

\subsection{Análise fatorial}

A abordagem estatística conferida pela utilização da análise fatorial é caracterizada pela verificação dos níveis de correlação de interdependência multivariada entre as variáveis. Sua utilização consiste na busca por consistências internas resultantes das correlações entre as variáveis presentes em uma amostra (Hair et al., 2014).

O propósito da análise fatorial consiste na redução da quantidade de variáveis, proporcionando que o número restante apresente elevado nível de consistência. Sua abordagem segue por duas vertentes: análise fatorial confirmatória (AFC), cuja característica inicia pelo entendimento prévio da relação de comportamento entre as variáveis, por meio de pesquisas prévias que identificaram a estrutura dos fatores presentes; e análise fatorial exploratória (AFE), quando não há um nível de conhecimento sobre tema abordado (Field, 2020; Hair et al, 2014).

Esta pesquisa iniciou com a utilização da análise fatorial exploratória (AFE), buscando identificar os níveis de conformidade e o comportamento dos dados em relação aos pressupostos do procedimento confirmatório da análise fatorial confirmatória (AFC). Como resultado, não foram identificadas inconsistências associadas aos aspectos de multi-colinearidade nem de singularidade, conforme sugerem os critérios de delineamento de distância de Cook e Mahalanobis.

Em seguida foi executada a Análise Fatorial Confirmatória (AFC) para verificar a adequabilidade do instrumento da pesquisa ao modelo conceitual adotado pela amostra do estudo. Os resultados dos índices de ajuste absoluto, incremental e parcimonioso foram considerados satisfatórios para verificar as características e o comportamento empírico das variáveis presentes no instrumento de pesquisa ao modelo da Escala de Valores Organizacionais - EVO, conforme mostra a Tabela 2. 
Research, Society and Development, v. 10, n. 6, e15110615449, 2021

(CC BY 4.0) | ISSN 2525-3409 | DOI: http://dx.doi.org/10.33448/rsd-v10i6.15449

Tabela 2: Guia para apuração dos resultados da EVO.

\begin{tabular}{lcc}
\hline \multicolumn{1}{c}{ Fatores } & Itens & Divisor \\
\hline Eficácia/eficiência & $05,07,10,12,13,25,28,31,32$ & 9 \\
Interação no trabalho & $01,02,03,04,06,08,09,11,15,36$ & 10 \\
Gestão & $14,17,23,24,29,37,38$ & 7 \\
Inovação & $19,20,22,30$ & 4 \\
Respeito ao funcionário & $16,18,21,26,27,33,34,35$ & 8 \\
\hline
\end{tabular}

Fonte: Autores (2021).

\section{Resultados e Discussão}

$\mathrm{O}$ modelo testado inicialmente apresenta as correlações e coeficientes associados à forma original da Escala de Valores Organizacionais - EVO. Os cinco Fatores são representados por elipses e os trinta e oito itens (ou variáveis) por retângulos associados a cada fator, conforme seus respectivos erros, em círculos. As setas unidirecionais representam a resposta de "causa" de cada sentença a cada Fator. 
Figura 1: Modelo Original da EVO.

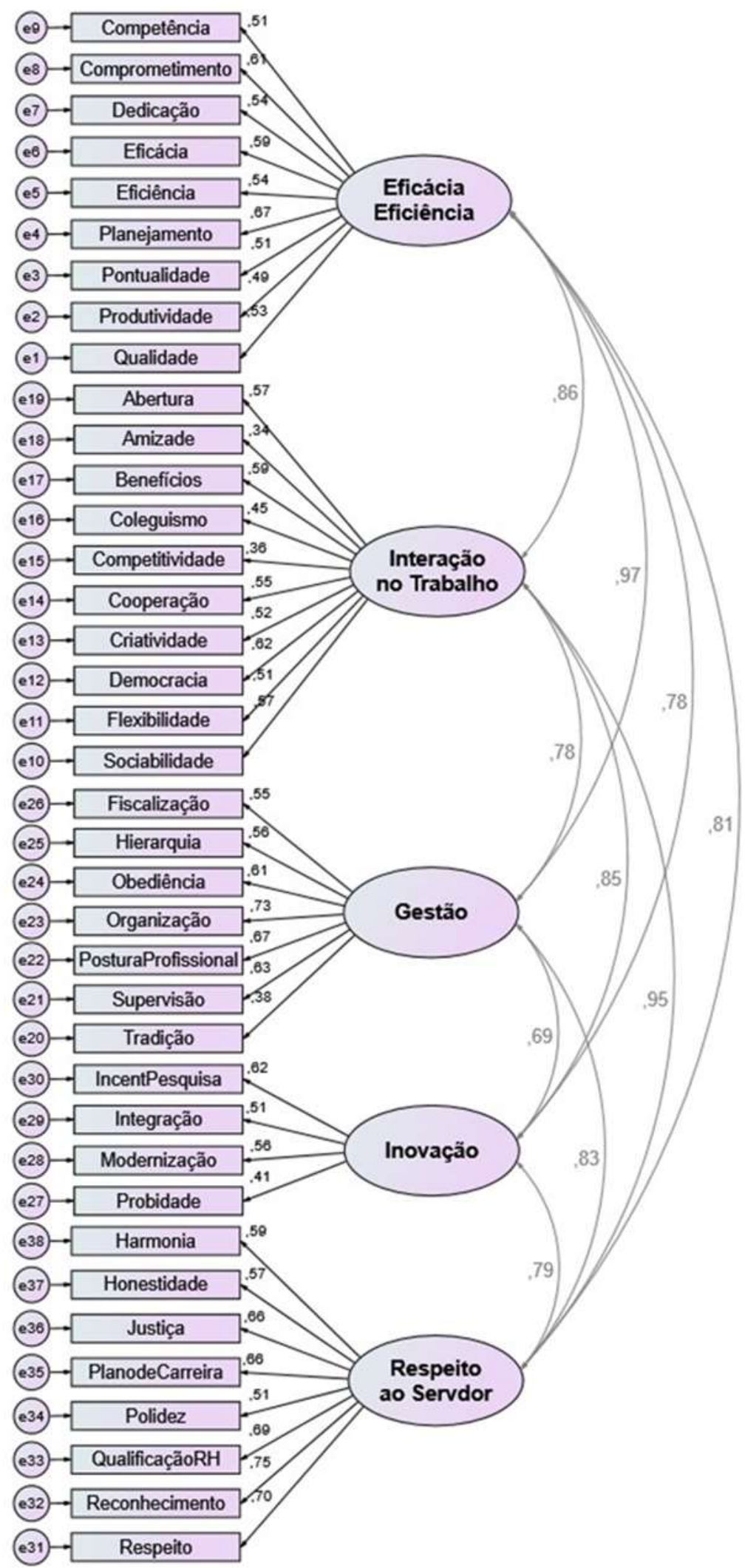

Fonte: Autores (2021).

Os Fatores estão latentes à manifestação verificada nas respostas das sentenças. No entanto, uma parte dessa manifestação não é resultante do respectivo fator, mas de causas não explicadas ou desconhecidas, representadas pelos erros. 
Assim, as setas são direcionadas dos erros para cada sentença. Por fim, as setas bi direcionadas entre os fatores indicam a pressuposição de correlação entre os fatores contemplados pelo modelo, conforme mostrado na Figura 1.

A Figura 2 apresenta o modelo ajustado da EVO, cujas características de agrupamento foram mantidas em cinco fatores, alterando somente a quantidade dos itens. As setas unidirecionais representam a resposta de "causa" de cada sentença a cada fator.

Figura 2: Modelo adaptado da EVO.

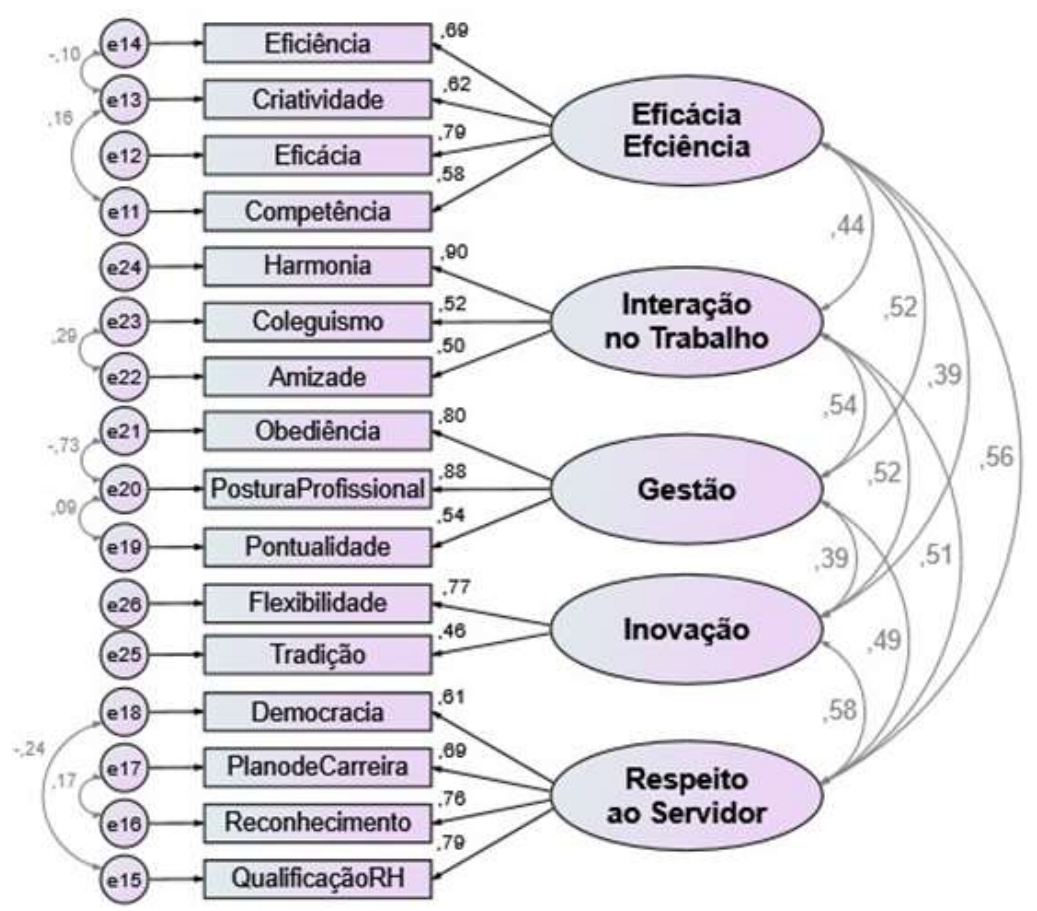

Fonte: Autores (2021).

Verificou-se que, dos 38 itens que compõem o instrumento original da EVO, 22 itens apresentaram baixos coeficientes padronizados e não seriam necessários para o contexto abordado nesse estudo - empresas familiares.

Após a exclusão dos itens citados, um novo modelo (adaptado) foi estruturado, conforme as características dos dados obtidos pelo instrumento de pesquisa, apresentando índices com valores extremamente aceitáveis. A Tabela 3 mostra o comparativo entre os resultados dos índices de ajuste obtidos pelos dois modelos apresentados. 
Tabela 3: Comparativo entre os resultados obtidos e os valores ideais para cada índice de ajuste.

\begin{tabular}{cccc}
\hline Medidas de Ajuste & Valor Ideal & Modelo Original & Modelo Adaptado \\
\hline$\chi^{2}$ & ---- & 2230,175 & 186,683 \\
gl. & ---- & 655 & 87 \\
$P_{\text {value }}$ & $<0,05$ & 0,000 & 0,000 \\
$\chi^{2}$ gl. & Entre 1 e 3 & 3,405 & 2,146 \\
GFI & $>0.90$ & 0,729 & 0,942 \\
SRMR & $<0.08$ & 0,075 & 0,047 \\
RMSEA & $<0.06$ & 0,082 & 0,056 \\
TLI & $>0,90$ & 0,692 & 0,923 \\
NFI & $>0,90$ & 0,64 & 0,904 \\
AGFI & $>0,90$ & 0,693 & 0,909 \\
CFI & $>0.95$ & 0,713 & 0,948 \\
\hline
\end{tabular}

Fonte: Autores (2021).

A identificação da qualidade dos ajustes apresentados na Tabela 3 foi verificada com base nas seguintes premissas:

a) Ajuste Absoluto - que representa a qualidade do modelo adaptado pelo pesquisador a partir dos dados observados. Foram considerados nesta pesquisa: $\chi^{2}$ (Qui-quadrado), GFI (Índice de qualidade de ajuste), RMR (Raiz do resíduo quadrático médio), RMSEA (Raiz quadrada média dos quadrados dos erros de aproximação);

b) Ajuste Incremental - que avalia como um modelo específico se ajusta de forma parcial para resultar em modelo adaptado. Foram considerados pela pesquisa: TLI (Índice de Tucker-Lewis), NFI (Índice de ajuste normalizado), AGFI (Índice ajustado de qualidade do ajuste);

c) Ajuste Parcimonioso - que complementa os dois tipos de ajustes anteriores e representa o nível de ajuste do coeficiente estimado a ser aplicado ao modelo adaptado. Objetiva tentar corrigir valores elevados de ajuste do modelo original, verificando a razão entre a parcimônia do modelo e sua qualidade. A pesquisa utilizou: $\chi^{2} /$ gl. (Qui-quadrado normalizado) e CVI (Índice de validação cruzada).

$\mathrm{Na}$ interpretação da análise de qualidade do ajuste do modelo verificou-se que todos os índices propostos atingiram os valores mínimos sugeridos na literatura, o que remete ao entendimento de que todos os índices considerados pela qualidade de ajuste global foram extremamente satisfatórios ao modelo adaptado EVO.

\section{Conclusão}

Em relação ao modelo original, diante do contexto delimitado pela pesquisa, o modelo adaptado EVO considera apenas 16 itens utilizados para identificar, com consistência, os valores mais relevantes para os profissionais das empresas com estrutura de gestão familiar abordada, conforme mostra a Tabela 4. 
Tabela 4: Síntese das variáveis presentes nos fatores dos modelos EVO original e adaptado.

\begin{tabular}{lcccc}
\hline \multicolumn{1}{c}{ Fatores } & Itens do Modelo Original & Divisor & Itens do Modelo Adaptado & Divisor \\
\hline Eficácia/eficiência & $05,07,10,12,13,25,28,31,32$ & 9 & $13,9,12,5$ & 4 \\
Interação no trabalho & $01,02,03,04,06,08,09,11,15,36$ & 10 & $16,4,2$ & 3 \\
Gestão & $14,17,23,24,29,37,38$ & 7 & $26,29,28$ & 3 \\
Inovação & $19,20,22,30$ & 4 & 15,38 & 2 \\
Respeito ao funcionário & $16,18,21,26,27,33,34,35$ & 8 & $33,34,26,11$ & 4 \\
\hline
\end{tabular}

Fonte: Autores (2021).

Os resultados indicam que o modelo adaptado, aplicado ao contexto da abordagem, fundamentou uma estrutura fatorial robusta, caracterizada pela consistência e qualidade dos índices obtidos (Tabela 3). Nesse sentido, o modelo adaptado apresentou validade e fidedignidade ao instrumento utilizado para análise das atribuições no aspecto empírico, respondendo positivamente ao questionamento central da pesquisa.

Sugere-se que futuras pesquisas sejam desenvolvidas e direcionadas a organizações com características similares, que atuem em diferentes segmentos de mercado. Dessa forma, será possível investigar em que grau a Escala de Valores Organizacionais - EVO pode funcionar como instrumento balizador na identificação de valores, motivação e expectativa dos profissionais que atuam em empresas de estrutura de gestão familiar, mensurando assim até que ponto determinado valor organizacional pode impactar em um ambiente de negócios.

Empresas familiares têm características próprias, que as diferem de outros tipos de organizações. Assim, acredita-se que, para a academia, este estudo poderá contribuir para um conhecimento mais profundo sobre os valores que predominam em organizações de cultura familiar, possibilitando reflexões para o trabalho em empresas deste gênero, uma vez que, conhecer o nível de engajamento das pessoas para com os valores e a cultura da organização familiar permitirá contribuir de maneira científica para o melhor conhecimento dessas organizações.

Pode-se ainda sugerir outros recortes nos resultados obtidos, como por exemplo, separando os três segmentos (indústria, comércio e serviços), uma vez que, para compreender a cultura de uma organização é necessário o conhecimento do contexto e da sociedade em que está inserida.

É provável ainda que as conclusões forneçam caminhos mais direcionados para empresas de cultura familiar de qualquer ramo de atividade, que estejam dispostas a repensar seus processos de gestão para atingir resultados melhores e organizações perenes.

\section{Referências}

Arayesh, M. B., Golmohammadi, E., Nekooeezadeh, M., \& Mansouri, A. (2017). The effects of organizational culture on the development of strategic thinking at the organizational level. International Journal of Organizational Leadership 6: 261-275.

Arif, M., Zahid, S., Kashif, U., \& Sindhu, M. I. (2017). Role of leader-member exchange relationship in organizational change management: Mediating role of organizational culture. International Journal of Organizational Leadership 6: 32-41.

Carvalho, O. e S. (2013). Valores organizacionais em instituições públicas brasileiras: percepções dos servidores em diferentes posições hierárquicas e tipos de entidade da administração indireta. RAM - Revista de Administração Mackenzie, S.P. 14(5), 74-103.

Chukwuka Z. C. (2016). Impact of organization culture and change on organizational performance. Global Journal of Applied, Management and Social Sciences 12:186-198.

Farrokhi, F., \& Mahmoudi, A. H. (2012). Rethinking Convenience Sampling: Defining Quality Criteria. Theory and Practice in Language Studies. 2(4), 784792.

Field, Andy (2020). Descobrindo a estatística usando o SPSS. Editora: Penso: (5a ed.).

Hair, J. F., Black, W. C., Babin, B. J., Anderson, R. E. \& Tatham, R. L. (2014). Análise Multivariada de Dados. (6a ed.), Bookman.

Handler, W. C. (1989). Methodological issues, and considerations in studying family businesses. Family Business Review. 2(3). $257-276$. 
Research, Society and Development, v. 10, n. 6, e15110615449, 2021

(CC BY 4.0) | ISSN 2525-3409 | DOI: http://dx.doi.org/10.33448/rsd-v10i6.15449

Lacerda, D. P. (2011). Cultura organizacional: sinergias e alergias entre Hofstede e Trompenaars. Revista de Administração Pública. 45(5), $1285-1301$.

Lewis, H. (1991). A question of values: six ways we make the personal choices that shape our lives. Harper Collins.

Marôco, João. (2014). Análise Estatística com o SPSS. (6a ed.), Pero Pinheiro.

Maximiano, A. C. A. (2005). Teoria geral da administração: da revolução urbana à revolução digital. (5a ed.), Atlas.

Motta F., Caldas, M. (1997). Cultura Organizacional e Cultura Brasileira. Atlas.

Nunnaly, J. C. (1967). Psychometric Theory. MacGraw-Hill Book Company.

Oliveira, A. F., \& Tamayo, A. (2004) Inventário de perfis de valores organizacionais. Revista de Administração da USP, 39(2), 129-140.

Ouchi, W. (1982). Teoria Z - como as empresas podem enfrentar o desafio japonês. Editora Fundo Educativo Brasileiro.

Rokeach, M. (1981) Crenças, atitudes e valores. Interciência.

Russo, G. M. (2012). Diagnóstico da Cultura Organizacional. Elsevier.

Schein, E.H. (2009) Cultura organizacional e liderança. Atlas.

Schwartz, S.H. (2019). A theory of cultural values and some implications for work. Applied Psychology: An International Review, 48, 23-47.

Tamayo, A. \& Gondim, M. D. G. C. (1996) Escala de valores organizacionais. Revista de Administração da USP, 31(2), 62-72.

Veiga, H. M. S. (2018) Comportamento Proativo: relações com valores organizacionais, estímulos e barreiras à criatividade nas organizações e normas sociais. Tese de Doutorado não publicada. Instituto de Psicologia, Universidade de Brasília.

Zaneti, G. B., Rocha, R. F. da, Fusco, J. P. A. \& Rodrigues, J. de S. (2014). Typology of companies' networks for competitive assessment: an application in higher education institutions. Business Management Review, 4(1), 76-76. 